hep-th/9704151

RU-97-12, CU-TP-823, IASSNS-HEP-97/24

\title{
Orbifold Resolution by D-Branes
}

\author{
Michael R. Douglas, ${ }^{1}$ Brian R. Greene, ${ }^{2, *}$ and David R. Morrison ${ }^{3, \dagger}$ \\ ${ }^{1}$ Department of Physics and Astronomy \\ Rutgers University \\ Piscataway, NJ 08855-0849 \\ mrd@physics.rutgers.edu \\ ${ }^{2}$ Departments of Physics and Mathematics \\ Columbia University \\ New York, NY 10025 \\ greene@math. columbia.edu \\ ${ }^{3}$ Schools of Mathematics and Natural Sciences \\ Institute for Advanced Study \\ Princeton, NJ 08540 \\ drm@math.duke.edu
}

We study topological properties of the D-brane resolution of three-dimensional orbifold singularities, $\mathbb{C}^{3} / \Gamma$, for finite abelian groups $\Gamma$. The D-brane vacuum moduli space is shown to fill out the background spacetime with Fayet-Iliopoulos parameters controlling the size of the blow-ups. This D-brane vacuum moduli space can be classically described by a gauged linear sigma model, which is shown to be non-generic in a manner that projects out non-geometric regions in its phase diagram, as anticipated from a number of perspectives.

April 1997

* On leave from Department of Physics, Cornell University, Ithaca, NY 14850.

$\dagger$ On leave from Department of Mathematics, Duke University, Durham, NC 27708-0320. 


\section{Introduction}

D-branes are interesting probes of topology and geometry - as explored in many recent works, spacetime is a derived concept from the D-brane perspective, emerging from nontrivial moduli spaces of D-brane world-volume gauge theories.

Orbifold resolution was the first non-trivial example of this phenomenon, studied in [1,2].3]. The classical Lagrangian of the probe theory is a projection of maximally supersymmetric super Yang-Mills theory, with Fayet-Iliopoulos terms controlled by twist sector moduli. In the two dimensional case $-\mathbb{C}^{2} / \Gamma$-it was shown that the classical gauge theory moduli space is the resolved orbifold, and the metric is computable. The result is a physical realization of the hyper-Kähler quotient construction of Kronheimer [ [4].

Here we demonstrate that an analogous procedure works for cyclic orbifold singularities in three complex dimensions, treating the simplest examples $\mathbb{C}^{3} / \mathbb{Z}_{3}$ and $\mathbb{C}^{3} / \mathbb{Z}_{5}$ explicitly. The world-volume theory has the equivalent of $d=4, \mathcal{N}=1$ supersymmetry, and the superpotential and D-terms are no longer related by supersymmetry. The mathematical counterpart of this statement is that the construction is not a hyper-Kähler quotient but rather a blow-up of a singular Kähler quotient. The superpotential defines the complex structure and the blow-up, while the D-terms define the periods of the metric (though the full metric depends on both data). The resulting theories are physical realizations of a generalization of Kronheimer's construction to $\mathbb{C}^{n} / \Gamma$ orbifold resolution, recently developed by Sardo Infirri [0, 6 .

In this note we discuss topological and qualitative aspects of the construction. We show that a generic deformation of the moduli from the orbifold point leads to complete resolution of the orbifold and produces a smooth metric.

At weak string coupling, where our analysis is directly applicable, D-branes provide a description of spacetime at short distances complementary to the closed string approach of [7,8.9]. In that approach, it was found that the Kähler moduli spaces of sigma model compactification form but a small subset of the possibilities. Linear sigma models - when available - provide a more general definition and reveal a rich phase structure of the moduli space including target spaces of different topology and non-geometric phases. Computations using mirror symmetry established that all of these phases are connected, and gave physical results suggesting a more geometrical picture even for the non-geometric phases.

By using D-brane gauge theory, we can study topological properties of orbifolds on length scales $r$ in the range $l_{p}^{11}<r<\sqrt{\alpha}^{\prime}$. The classical physics of these probe theories 
turns out to also be describable by gauged linear sigma models, and a priori, the moduli space of these linear sigma models could again have numerous phases. We show, though, that the D-brane linear sigma models are not generic in that they only probe part of the linear sigma model moduli space. We present evidence that this subspace is nothing but the "partially enlarged Kähler moduli space," every point of which has a geometrical interpretation. Thus D-branes appear to directly project out the non-geometric phases.

We believe that this D0-brane description remains qualitatively correct even at strong string coupling, following the lines of [10,11,12]. (This issue will be discussed further in [13].) If so, our results confirm an idea of Witten that in $M$ theory only geometric phases appear [14.

In section 2, we review some relevant aspects of the stringy description of the moduli space of Calabi-Yau compactification. In section 3 we discuss the D-brane construction. In section 4 we derive some of the qualitative features the moduli spaces, using the gauged linear sigma model description. Section 5 gives conclusions and some open questions.

\section{Quantum geometry of Calabi-Yau manifolds}

At present there are two limits near which one can understand the quantum moduli space of Kähler structures on a Calabi-Yau manifold: weakly coupled Ila (or Ilb) string theory and $\mathrm{M}$ theory. As the explicit analysis we carry out is justified in the weak string coupling limit, we first describe our work in the Ila framework; subsequently, we comment on its possible relevance in $\mathrm{M}$ theory, assuming unexpected surprises do not arise when extrapolating to strong coupling.

The starting point is a classical moduli space of metrics on a Calabi-Yau manifold $M$. This is locally a product of a space of complex structures $\mathcal{M}_{C}$ and complexified Kähler forms $\mathcal{M}_{K}$. Classically, $\mathcal{M}_{K}$ is a complexification of the Kähler cone $\mathcal{M}_{K c}$, the subset of $H^{2}(M, \mathbb{R})$ for which the volumes of cycles are positive. As such, it appears to have boundaries, each of which requires physical explanation. A priori these explanations would be expected to involve quantum effects, and this is the subject of quantum geometry.

For our present purpose it is worthwhile to build up to the quantum corrected version of $\mathcal{M}_{K c}$ in two steps. First, one must augment the complexification of $\mathcal{M}_{K c}$ to $\mathcal{M}_{K c}^{\text {p.e. }}$, the partially enlarged Kähler moduli space, which consists of the classical complexified Kähler cones of all Calabi-Yau manifolds related to $M$ by flops along rational curves, glued along common faces. Second, realizing that this does not exhaust the full moduli space [7,8], 
one must augment $\mathcal{M}_{K c}^{\text {p.e. }}$ by gluing on additional regions (or "phases") which arise when higher dimensional subspaces of $M$ or its birational cousins are shrunk to points.

Quantum corrections can also change the boundaries between different phases. In weakly coupled string theory, these changes are by effects of order $\alpha^{\prime}$. Thus it may be that even in a phase in which a cycle shrinks to a point, its observed volume is non-zero.

The final result is sometimes called the enlarged nonlinear sigma model Kähler moduli space $\mathcal{M}_{K c}^{\text {e.n. }}$, though it is already a good description of $\mathcal{M}_{K}$ at weak string coupling.

There are two approaches to carrying out the second step. One can seek a mirror manifold $W$ of $M$ and study its complex structure moduli space using standard techniques from algebraic geometry, or one can seek a gauged linear sigma model description of $M$. Each has its advantages and disadvantages. The linear sigma model approach allows us to physically identify all of the additional regions in $\mathcal{M}_{K c}^{\text {en. }}$. These are usually called "non-geometrical phases" since they are not interpretable in terms of smooth Calabi-Yau sigma models. Instead, they are most naturally described in terms of more abstract field theories such as orbifolds, Landau-Ginsburg models, and various hybrid combinations. However, unlike the mirror symmetry approach, the linear sigma model does not construct

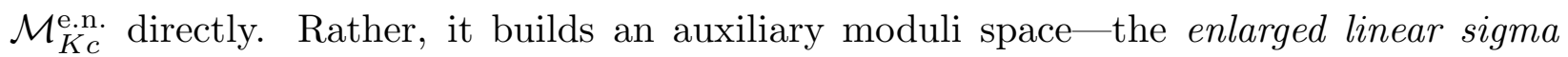
model moduli space $\mathcal{M}_{K c}^{\mathrm{e} .1}$. The latter is discussed in detail in [8] as well as [7, 9, 15, 16]; it fills out all of $H^{2}(M, \mathbb{C})$, for example. The space $\mathcal{M}_{K c}^{\mathrm{e} . \mathrm{l}}$ must then be subjected to a

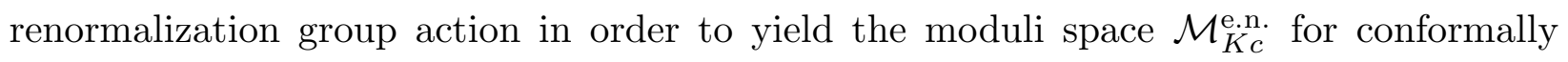
invariant physical models. In other words, the linear sigma model parameters mapped out by $\mathcal{M}_{K c}^{\text {e.l. }}$ are secondary constructs which can be used to label universality classes of physical string models; their primary physical counterparts, however, are the parameters which arise upon passing to the conformal limit.

Of course, regardless of which approach one follows, the final form for $\mathcal{M}_{K c}^{\mathrm{e} . \mathrm{n}}$ is the same. The essential properties of $\mathcal{M}_{K c}^{\mathrm{e} . \mathrm{n}}$ for our present concerns are three-fold. First, $\mathcal{M}_{K c}^{\mathrm{e} . n .}$ has numerous phase regions, some of which have a manifest geometric interpretation in terms of Calabi-Yau nonlinear sigma models, while others do not. The boundaries in $\mathcal{M}_{K c}^{\text {e.n. }}$ are not sharp phase transitions but rather delineate the edges of perturbative convergence in each region. One can pass from region to region by analytic continuation. Second, the boundaries of the classical geometrical moduli space $\mathcal{M}_{K c}^{\text {p.e. }}$ get shifted by terms of order $\alpha^{\prime}$ in the quantum corrected moduli space $\mathcal{M}_{K c}^{\text {e.n. }}$. Third, $\mathcal{M}_{K c}^{\text {e.n. }}$ appears to be a subset of the classical moduli space $\mathcal{M}_{K c}^{\text {p.e. }}$. This inclusion has not been proven, but strong circumstantial evidence has been presented in [9]. Assuming it to be true, we learn that every point in 


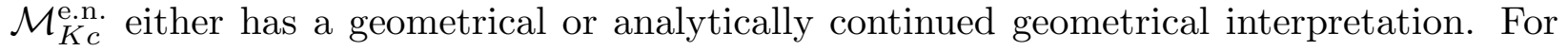
instance, a Landau-Ginsburg, hybrid or orbifold phase can be interpreted as the analytic continuation of a smooth Calabi-Yau sigma model, beyond the realm of sigma model perturbation theory, (i.e., to distance scales on the order of $\sqrt{\alpha^{\prime}}$ ).

As D-branes are natural probes of sub-stringy distance scales, it is important to understand the geometrical structure they sense at such points in moduli space. D-brane geometry arises in a different manner from the traditional fundamental string approach. Whereas a classical background geometry is generally introduced in fundamental string theory and then subjected to various corrections, D-brane geometry emerges from the study of the D-brane vacuum moduli space. In general this space also undergoes quantum corrections, but in the particular case of D0-branes at weak string coupling, these are suppressed. Thus the geometry can be accessed through a classical study of the vacuum structure of a world volume super-Yang-Mills theory.

In our present study of orbifold resolution, this world-volume theory will turn out to be classically describable as a gauged linear sigma model, which would seem to fit well with the fundamental string description above. However, closer inspection reveals a crucial difference: the linear sigma model describing D-branes is not the one usually used to study fundamental strings. Now in the fundamental string context, it did not really matter which linear sigma model was used as a starting point. Only properties of the conformally invariant limit were observable, and a central ingredient in this analysis was the associated use of the renormalization group to pass to the conformally invariant limit. It was this process that gave an analytically continued geometrical interpretation to the "non-geometric" phases. For D-branes there is no analogous step. If the D-brane linear sigma model analysis were to yield non-geometric phases, we would have to live with them.

In the following sections we shall see that the D-brane linear sigma model differs from that arising from fundamental strings in just the right way to yield a compatible spacetime interpretation while avoiding the non-geometric phases. At least in the orbifold examples we study, spacetime at short distances as probed by D-branes agrees with the analytically continued geometry probed by fundamental strings. In a novel fashion, D-branes avoid the non-geometrical phases.

What happens if we try to extrapolate this picture to large string coupling? In the $\mathrm{M}$ theory limit, the string scale is far below the eleven-dimensional Planck scale, leading to the conjecture that additional quantum effects vanish [14]. Specifically, in [14] it was argued that the non-geometric phases in the type IIa vector multiplet moduli space are 
squeezed to zero size. What this means is that the geometrical phases contained in $\mathcal{M}_{K c}^{\text {e.n. }}$ - the conformal limit of $\mathcal{M}_{K c}^{\text {p.e. }}$ - expand to fill out the region delineated by the classical counterpart, $\mathcal{M}_{K c}^{\text {p.e. }}$, while the non-geometrical phases are flattened out to constitute the boundaries. In other words, all physical models again have a geometrical interpretation, except now we do not even have to perform analytic continuation to find it. This is precisely in accord with our D-brane picture which avoids non-geometrical phases from the word go. In this sense, the strong coupling extrapolation of our analysis confirms part of the M-theory picture of [14]. It would very interesting to extend this analysis to the other "non-geometrical" phases such as Landau-Ginsburg models and hybrids and see if the same conclusion follows.

An important conclusion of [9] is that conformal field theory orbifold points have a nonzero $B$-field. This implies, as pointed out in [17], that wrapped D2-branes and D4branes have nonzero mass at the deep interior point of an orbifold phase. On the other hand, there are boundary points of the orbifold phase where the quantum volume of cycles associated with the singularity do vanish, and it is here that wrapped D-branes become massless. In the strong string coupling limit, as the orbifold phase gets squeezed, the conformal field theory orbifold point and the singular boundary point coincide. 1 The wrapped D-branes at the non-singular orbifold point should become massless in this limit. We can explicitly see this in our D-brane analysis by identifying wrapped states with "fractional" D-brane configurations. We will show that the masses of these states go to zero as $1 / g_{s}$ in the strong string coupling limit.

\section{3. $\mathbb{C}^{3} / \Gamma$ orbifold compactification}

We consider a type II string theory compactified on $\mathbb{C}^{3} / \Gamma$, with $\Gamma$ a $\mathbb{Z}_{n}$ subgroup of $S U(3)$. We take $n$ odd to get an isolated singularity. It is well known that these orbifolds can be resolved to smooth spaces with $b_{3}=0$; in the simplest example of $\mathbb{C}^{3} / \mathbb{Z}_{3}$ this is just a blow-up replacing the singularity with a $\mathbb{P}^{2}$. As it happens, in the general case $b_{2}=b_{4}=(n-1) / 2$.

We take complex coordinates $Z^{i}$ and a generator $g$ of $\mathbb{Z}_{n}$ labeled by three integers $\left(a_{1}, a_{2}, a_{3}\right)$ with $a_{1}+a_{2}+a_{3} \equiv 0(\bmod n)$. It acts as $Z^{i} \rightarrow \omega^{a_{i}} Z^{i}$ with $\omega \equiv \exp 2 \pi i / n$. Thus the unbroken $d=4$ supersymmetry will be $\mathcal{N}=2$ in the closed string sector, and $\mathcal{N}=1$ on the D-branes.

1 This can also seen by analyzing the orbifold point directly in M theory compactified on the product of $S^{1}$ with a three-dimensional orbifold [18,19]. 


\subsection{Closed string spectrum}

The closed string sector has $n-1$ twist sectors. In type II theory each contains a complex NS-NS field $\phi_{k}$, and a complex R-R field making up a hyper (IIb) or vector (IIa) multiplet. There is a reality condition [20]

$$
\phi_{n-k}=\phi_{k}^{*}
$$

leaving $(n-1) / 2$ complex NS-NS fields. These are the fields which in the large volume limit become the complexified Kähler forms $B+i J$ on the resolution. The $\mathrm{R}-\mathrm{R}$ fields in the $k^{\text {th }}$ twisted sector are a complex bispinor in $d=4$ satisfying the same reality condition.

These decompose into complex $p$-form potentials $\tilde{C}_{k}^{(p)}$, where the GSO projection enforces two properties (see for example [21,22]): first, $p$ must be even for $\mathrm{Ilb}$ and odd for Ila, and second, $d \tilde{C}_{k}^{(p)}=i * d \tilde{C}_{k}^{(2-p)}$. These $p$-form potentials correspond in the large volume limit to integrals of the bulk R-R fields over various cycles. In Ilb, these are $\int_{\Sigma_{2}} C^{(2)}$ and $\int_{\Sigma_{4}} C^{(4+)}$ (since the fields $\int_{\Sigma_{2}} C^{(4+)}$ are redundant due to the self-duality of $C^{(4+)}$ ), while in IIa, they are $\int_{\Sigma_{2}} C^{(3)}$ (whose duals are of the form $\int_{\Sigma_{4}} C^{(5)}$ ).

The type I spectrum is the subsector of type Ilb surviving the $\Omega$ (world-sheet twist) projection. This removes the $B$ moduli, leaving $k$ NS-NS moduli to control the real Kähler forms, and $k$ R-R scalars.

\subsection{D-brane theory}

On physical grounds, we expect type II compactification on the large volume limit of a Calabi-Yau to contain the same $\mathrm{D} p$-branes as the uncompactified theory, with D-branes wrapped about supersymmetric cycles giving BPS states.

As curvatures approach the string scale, stringy corrections to the world-volume Lagrangians of these branes could become important. For example, a single D0-brane will have a world-volume kinetic term incorporating a metric $g_{\mu \nu}$ which, although Ricci-flat in the small curvature limit, a priori need not be, just as the superstring sigma model metric satisfies a modified Einstein equation with corrections of order $O\left(\alpha^{\prime 3}\right)$. The D0-brane metric need not be exactly equal to the string metric and at this writing explicit results for these metrics have not been obtained. However it seems likely that such corrections are present.

Conformal field theory provides a more general language for compactification, and Dbranes in this general context have been discussed in [23. It is a very interesting question 
whether moduli spaces of superconformal boundary conditions are always similar to the geometric moduli spaces of the sigma model D-branes.

Here we discuss D-branes defined on an orbifold and related theories obtained by adding twisted sector moduli with small coefficients, following the approach of [1] in two complex dimensions. The basic results we rely on are, first, an analysis of world-sheet consistency conditions which shows that the orbifold prescription of closed string theory also applies to open string theory, with all new "open string twisted sectors" provided by adding images of the original D-branes.2 Second, analyticity of conformal perturbation theory around the orbifold point guarantees that the D-brane world-volume Lagrangian will be the projected flat space Lagrangian with corrections analytic in the blowup parameters.

Thus a Dirichlet $p$-brane at a point in $\mathbb{C}^{3} / \Gamma$ will be defined as the quotient of the theory of $n$ Dirichlet $p$-branes in $\mathbb{C}^{3}$ by a combined action of $\Gamma$ on $\mathbb{C}^{3}$ and the D-brane index (Chan-Paton factor). A single Dirichlet $p$-brane is described by taking the ChanPaton factors in the regular representation of $\Gamma$. It is also possible to consider other representations as in [11] (intuitively, leaving out some of the images) as we discuss below.

The D-brane theory on $\mathbb{C}^{3}$ is dimensionally reduced $d=4, \mathcal{N}=4$ supersymmetric gauge theory with gauge group $U(n)$. The generator $g$ now acts as above on $\mathbb{C}^{3}$ and on the Chan-Paton factors as the regular representation, $\gamma(g)_{i j}=\delta_{i j} \omega^{i}$. The gauge field projection is

$$
A_{i, j}=\omega^{i-j} A_{i, j}
$$

leaving the subgroup $U(1)^{n}$ unbroken.

The complex positions are the bosonic components of chiral multiplets. They must satisfy

$$
X_{i, j}^{\mu}=\omega^{i-j+a_{\mu}} X_{i, j}^{\mu}
$$

Thus, the non-zero fields take the form $X_{i, i+a_{\mu}}^{\mu}$, and each such field is charged under two $U(1)$ 's. All fields are uncharged under the diagonal $U(1)$.

The action on the fermions is determined the same way, but now taking $g$ to act on spinors. For $\Gamma \subset S U(3)$, the $\mathcal{N}=4$ gaugino will decompose in the $\mathcal{N}=1$ theory into a singlet (partners of (3.2)) and triplet (partners of (3.3)). For $\Gamma \not \subset S U(3)$, the projection will generally lead to a non-supersymmetric theory.

2 The essential features of this analysis can be found in [24] and [1]; related issues are treated in [25] and references therein. We also thank T. Banks and M. Berkooz for discussions on this topic. 
This spectrum can be described by a quiver diagram as in [1], with a node corresponding to each $U(1)$ factor of the gauge group, and an oriented link for each chiral multiplet (as in figure 1).

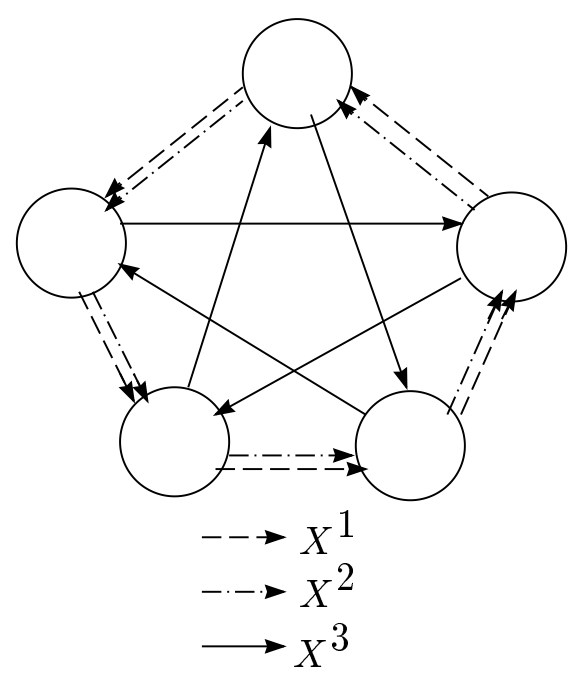

Fig. 1: Quiver diagram for $n=5$ and $\vec{a}=(1,1,3)$.

The Lagrangian is simply the $\mathcal{N}=4$ Lagrangian evaluated on fields satisfying the projection, including a Fayet-Iliopoulos term with coefficient $\zeta_{i}$ associated to the $i^{\text {th }} U(1)$, for each $i$. Supersymmetric vacua will satisfy D-flatness conditions,

$$
\zeta_{i}=-\sum_{\mu=1}^{3}\left|X_{i, i+a_{\mu}}^{\mu}\right|^{2}+\left|X_{i-a_{\mu}, i}^{\mu}\right|^{2},
$$

with $\sum \zeta_{i}=0$ required for supersymmetric vacua to exist.

The superpotential is the reduction of the $\mathcal{N}=4$ superpotential,

$$
\begin{aligned}
W & =\operatorname{Tr}\left[X^{1}, X^{2}\right] X^{3} \\
& =\sum_{i} X_{i, i+a_{1}}^{1} X_{i+a_{1}, i+a_{1}+a_{2}}^{2} X_{i+a_{1}+a_{2}, i}^{3}-X_{i, i+a_{2}}^{2} X_{i+a_{2}, i+a_{2}+a_{1}}^{1} X_{i+a_{2}+a_{1}, i}^{3} .
\end{aligned}
$$

The vacuum satisfies $\partial W / \partial X_{i}^{\mu}=0$ which produces the matrix equations

$$
\left[X^{\mu}, X^{\nu}\right]=0
$$


or in components,

$$
\begin{aligned}
X_{i, i+a_{1}}^{1} X_{i+a_{1}, i+a_{1}+a_{2}}^{2} & =X_{i, i+a_{2}}^{2} X_{i+a_{2}, i+a_{2}+a_{1}}^{1} \\
X_{i, i+a_{2}}^{2} X_{i+a_{2}, i+a_{2}+a_{3}}^{3} & =X_{i, i+a_{3}}^{3} X_{i+a_{3}, i+a_{3}+a_{2}}^{2} \\
X_{i, i+a_{3}}^{3} X_{i+a_{3}, i+a_{3}+a_{1}}^{1} & =X_{i, i+a_{1}}^{1} X_{i+a_{1}, i+a_{1}+a_{3}}^{3}
\end{aligned}
$$

Although naïvely there are $3 n-3$ conditions, which combined with the $n-1$ quotients by $U(1)$ would eliminate the (Higgs branch) moduli space, these conditions are not transverse to one another and thus the solution to (3.7) can be a non-trivial variety, which we denote by $\mathcal{N}_{\vec{a}}$. For example, it is easy to verify that with no Fayet-Iliopoulos terms, the Higgs branch $\mathcal{N}_{\vec{a}} / / U(1)^{n-1}$ coincides with $\mathbb{C}^{3} / \mathbb{Z}_{n}$, e.g. by taking $X^{\mu}=x^{\mu} \gamma(g)^{a_{\mu}}$.

The Fayet-Iliopoulos coefficients $\zeta_{i}$ control the metric on moduli space, and as in [1] this means they should be controlled by twisted moduli. The computation of these couplings in the appendix of [1] can be extended to this case. The main point is the rôle of the Chan-Paton twist $\gamma(g)$ in the amplitude - a coupling to the sector twisted by $g^{k}$ corresponds to a world-sheet amplitude with an insertion of $\gamma\left(g^{k}\right)$ at the end-point of the cut produced by the closed string twist field. The couplings between the R-R fields and gauge field strengths on the branes are then easy to compute, while the Fayet-Iliopoulos terms are the supersymmetric partners. This leads (in the example of the IIb D1-brane, with the other $\mathrm{D} p$-branes being analogous) to couplings of the form

$$
\sum_{k=1}^{n-1} \int d^{2} x \tilde{C}_{k}^{(2)} \operatorname{Tr} \gamma\left(g^{k}\right)+\tilde{C}_{k}^{(0)} \operatorname{Tr} \gamma\left(g^{k}\right) \mathcal{F}+\phi_{k} \operatorname{Tr} \gamma\left(g^{k}\right) \mathcal{D}
$$

where $\mathcal{F}$ is the matrix of gauge field strengths (satisfying the projection $\gamma(g) \mathcal{F} \gamma(g)^{-1}=\mathcal{F}$ ), and $\mathcal{D}$ the corresponding matrix of auxiliary fields. Since $\gamma\left(g^{k}\right)_{i i}=\omega^{i k}$, the coupling involves a discrete Fourier transform. In the $\mathbb{Z}_{3}$ example, we have $\phi_{2}=\phi_{1}^{*}$ and the coefficients of the D-terms become

$$
\zeta_{i}=\omega^{i} \phi_{1}+\omega^{2 i} \phi_{1}^{*}
$$

which satisfy $\sum \zeta_{i}=0$ and see both real and imaginary parts of $\phi_{1}$, in other words, both $J$ and $B$. 


\section{3. "Fractional branes" and wrapped branes}

As discussed in [11], world-sheet consistency conditions do not require the image D-branes to fill out the regular representation of $\Gamma$. Even if we start with the regular representation, when the images sit at the fixed point, there are flat directions which separate them in the non-compact space (the Coulomb branch) [2]. Thus if we claim that the orbifold resolution is geometric, we must find a geometric interpretation for these states.

Let us consider the world-volume theories associated with the one-dimensional representations of $\Gamma$. Specifically, for each $k, 1 \leq k \leq n$, we can consider the representation $\gamma^{(k)}$ defined by $\gamma^{(k)}(g)_{i j}=\delta_{i k} \delta_{j k} \omega^{k}$. The orbifold projection removes the transverse coordinates and the only surviving matter is the position in the non-compact space, so this theory describes an object bound to the fixed point. Its mass and charge can be determined by doing world-sheet computations of the one-point function of the metric or R-R string vertex operators, and the leading term comes from a disk diagram with boundary on the D-brane. The dependence on the representation $\gamma$ is the factor $\operatorname{Tr}\left(\gamma^{i}\right)$ in the $i^{\text {th }}$ twisted sector as in (3.8).

Since each image couples equally to the metric, in conventions where a conventional (regular representation) brane has mass $1 / g_{s}$, a single image has mass $1 / n g_{s}$. This is exact at the orbifold point, while turning on background fields can modify the mass, as we discuss shortly. Similarly, in conventions where the R-R charge of a conventional brane is 1 , the brane associated with the $k^{\text {th }}$ representation has charge $\omega^{i k} / n$ for the $\mathrm{R}-\mathrm{R}$ field in the $i^{\text {th }}$ twisted sector. This is the same discrete Fourier transform which appears in the gauge field couplings and it is simpler to think of the $k^{\text {th }}$ representation as associated with the $k^{\text {th }}$ $U(1)$ in the quiver diagram, and work with R-R fields in the Fourier transform basis as well. The scalars in this basis control Fayet-Iliopoulos parameters; as above the $k^{\text {th }}$ and $(n-k)^{\text {th }}$ scalar control $B$ and $J$ for the $k^{\text {th }}$ two-cycle. Thus the $k^{\text {th }}$ brane is charged under the R-R fields associated with the $k^{\text {th }}$ (or $\left.(n-k)^{\text {th }}\right)$ two-cycle and four-cycle. (This discussion is closely related to the generalized McKay correspondence which holds for arbitrary finite subgroups of $\mathrm{SU}(3)$ 26,27.)

The natural interpretation of all this is that the $k^{\text {th }}$ "fractional brane" is actually a Dirichlet $(p+\ell)$-brane, wrapped around an $\ell$-cycle implicit in the orbifold theory, to produce a D $p$-brane in the lower dimensional theory (or a bound state of such branes) [2, 11]. Further confirmation of this can be found by considering the supersymmetric partners 
of these couplings. For example, turning on the Fayet-Iliopoulos parameter $\zeta_{i}$ for the $U(1)$ associated with the brane increases its mass by $\zeta_{i}^{2} / n g_{s}$, the only term in the D-term potential surviving the projection. On the other hand, the brane still has the same fermion zero modes and corresponds to an object in the same supermultiplet, so the result must be a mass for the brane consistent with the central charge formula.

In gauge theory terms, turning on $\zeta_{i}$ eliminates the supersymmetric vacua on the Higgs branch; however the supersymmetry breaking is of a trivial form, a constant shift of the Hamiltonian. This must be associated with a central charge and it would be interesting to make this precise.

These results for the masses are controlled at weak string coupling and for small blow-up parameter $|\zeta|<<\alpha^{\prime}$. For $|\zeta| \sim \alpha^{\prime}$, we would expect them to be modified by corrections to the D-brane world-volume Lagrangian analytic in $\zeta / \alpha^{\prime}$, defined in principle by string world-volume computation. The phase boundary between orbifold and non-linear sigma model phases [7] might find a counterpart here in the domain of convergence of this expansion.

In the $\mathbb{Z}_{3}$ example, the three elementary wrapped branes have charges $(1,0)$, $\left(-\frac{1}{2}, \sqrt{3} / 2\right)$ and $\left(-\frac{1}{2},-\sqrt{3} / 2\right)$ under the real and imaginary parts of $\tilde{C}_{1}^{(p)}$. In a more natural charge basis, the charges of the three states are $\left(\begin{array}{ll}1 & 0\end{array}\right),\left(\begin{array}{ll}0 & \frac{1}{2}\end{array}\right)$ and $\left(-1-\frac{1}{2}\right)$; not having computed the kinetic term for $\tilde{C}_{1}^{(p)}$, the normalization of these charges is undetermined, so we might as well use this basis.

In IIa theory, we could consider "fractional" zero-branes; what states are these connected to in the large radius limit? Zero-branes can come from wrapped D2-branes, wrapped D4-branes or bound states of these objects. These objects are electrically and magnetically charged (respectively) under the $U(1)$ associated with the two-cycle. Evidently the first object is the wrapped two-brane and the second two are wrapped fourbranes; the electric charge of the wrapped four-branes comes from the expectation value for $B$ and the coupling $\int C^{(2)} \wedge(F-B)$. The symmetry between the two wrapped four-branes fits well with the known value $B=\frac{1}{2}$ at the orbifold point 9 ].

The masses $1 / n g_{s}$ of the elementary fractional branes implicitly determine the Kähler modulus $A=\int B+i J$ at the orbifold point, as well as its special geometry partner $\mathcal{F}^{\prime}(A)$ determining the masses of magnetically charged states. For example, the mass spectrum of the $\mathbb{C}^{3} / \mathbb{Z}_{3}$ case suggests that the gauge coupling is $\tau=e^{2 \pi i / 3}$, which is natural as the R-R gauge field coupling does not depend on $g_{s}$. 
All of these masses can also be determined by mirror symmetry techniques [9, 28], though not all of these predictions have been made explicit yet; for example the masses of wrapped four-branes (currently under investigation). Completing this comparison, besides confirming these identifications, would give a microscopic explanation of the masses of wrapped branes. Symmetries which can only exist in quantum geometry, such as the $\mathbb{Z}_{3}$ acting on the wrapped two-branes and four-branes of our example, would also be given an explicit microscopic picture.

Besides the elementary branes associated with one-dimensional representations, the general theory of this form can contain bound states at threshold, which will correspond to wrapped branes of higher degree. Such branes are expected to exist at large volume [14] and it will be interesting to identify these as well.

\subsection{Further corrections to the world-volume Lagrangian}

In the case of $\mathbb{C}^{2} / \Gamma$ considered in [1], supersymmetry determined the kinetic term in the D-brane world-volume Lagrangian, preventing other dependence on the twist fields $\phi_{i}$. (See 112] for further discussion, especially of the case of several D0-branes.) In the present case, this is no longer true. The configuration space can have a non-trivial metric, as long as it is asymptotically flat and reduces to the flat metric for $\phi_{i}=0$. In principle, it can be determined at weak string coupling by explicit world-sheet computation, again along the lines of [1].

The metric could also get corrections in the string loop expansion. For general $p$ branes, these could be important even at weak string coupling, due to world-volume IR effects (i.e., renormalization). However, for D0-branes, the dynamics are governed by a nonsingular quantum mechanical theory of heavy objects, and as such, IR effects are controlled by the masses of the world-volume degrees of freedom, which in turn are determined by the blow-up parameters $\zeta$. Thus the loop expansion will be controlled by the parameter $g_{s} / \zeta^{3 / 2}$. For blow-ups which are large compared to the eleven-dimensional Planck scale but small compared to the string scale, these effects are small, justifying the classical description.

On the other hand, these corrections are uncontrolled in the strong coupling limit, and the bare metric must be regarded as undetermined. Thus, the definition of $\mathrm{M}$ theory on an orbifold advocated in [11], as the strong coupling limit of the D0-brane theory (which if interpreted following the proposal of [10] could give a complete definition of $\mathrm{M}$ theory in this background), is not fully explicit for $\mathbb{C}^{3} / \Gamma$. 
This is an important point because the simplest version of the construction-namely, to use the flat metric for the D-brane gauge theory configuration space - appears to disagree with physical expectations. M theory for low energy processes on manifolds of small curvature $\left(R \ll 1 / l_{p l}^{2}\right)$ reduces to supergravity, and thus one might expect the low energy configuration space in the problem at hand to have a Ricci-flat metric. The classical treatment (which can be justified for D0-branes in this regime) produces the metric as a Kähler quotient, which on general grounds has no reason to be Ricci-flat. Indeed, Sardo Infirri has argued that in the example of $\mathbb{C}^{3} / \mathbb{Z}_{3}$, the relevant metric is not Ricci-flat [5].

One possible resolution of this discrepancy is that the bare metric might not be flat. 3 Another is that bound states of large numbers of D0-branes see an effective metric which is Ricci flat. These issues will be discussed in [13] and [30], but for present purposes, when we make statements about $\mathrm{M}$ theory, we are assuming that some form of the D0-brane system defined here, in particular with a non-singular kinetic term, is a correct representation of the strong coupling limit. This will allow us to make certain topological and even geometric statements.

\section{Geometrical interpretation}

In this section we shall consider the topological properties of the D-brane vacuum moduli space. Since sub-string scale spacetime emerges from this moduli space, we anticipate that the construction just outlined is geometrically interpretable as $\mathbb{C}^{3} / \Gamma$ with the smoothing of its singularities being controlled by the Fayet-Iliopoulos parameters. As we reviewed, previous studies have shown [7, [8], though, that perturbative string theory probes a rich phase structure associated with an orbifold background. For various choices of the blow-up parameters, the resulting configuration can be any of a number of birationally equivalent but topologically distinct smooth Calabi-Yau spaces, partially resolved orbifolds, Landau-Ginzburg orbifolds, or hybrids which mix these possibilities. From the perspective of perturbative string theory all of these distinct backgrounds smoothly join on to one another as the Fayet-Iliopoulos parameters are varied. By interpreting the D-brane vacuum moduli space as an effective spacetime background, we will be able to determine the fate of these phases at ultra-short distances.

The classical D-brane moduli space, for fixed values of the Fayet-Iliopoulos parameters, is obtained by imposing the superpotential (3.7) and D-term constraints (3.4), and

3 A similar phenomenon has recently been observed in an analogous context [29. 
quotienting by the residual $U(1)^{n-1}$ gauge symmetry group. Our analysis of this moduli space will be based on the observation - also made by Sardo Infirri [6] - that this space can also be described using toric geometry, or equivalently, described as the classical moduli space for an abelian gauged linear sigma model. The toric description has the pleasant feature of treating the superpotential and D-term constraints in a more-or-less uniform manner.

\subsection{A toric presentation}

To make the translation into a toric description, we begin by solving (3.7) on a dense open subset. Let us choose our generator of $\Gamma=\mathbb{Z}_{n}$ to have $a_{3}=-1$ and define $a=a_{1}$ and $b=a_{2}$. Defining $x_{i}=X_{i, i+a_{1}}^{1}, y_{i}=X_{i, i+a_{2}}^{2}$ and $z_{i}=X_{i, i+a_{3}}^{3}$, we note that we can use (3.7) to solve for $2 n-2$ of the variables in terms of the remaining set of $n+2$. In particular, we can solve for $x_{k}$ in terms of $x_{k-1}$ and the $z_{i}$ as

$$
x_{k}=\frac{z_{k}}{z_{a+k}} x_{k-1}
$$

Iterating this produces

$$
\begin{aligned}
x_{k} & =\frac{z_{1} \ldots z_{k}}{z_{a+1} \ldots z_{a+k}} x_{0} \\
& =\frac{z_{1} \ldots z_{k} z_{1} \ldots z_{a}}{z_{1} \ldots z_{a+k}} x_{0} .
\end{aligned}
$$

with all indices taken modulo $n$. Similarly,

$$
y_{k}=\frac{z_{1} \ldots z_{k} z_{1} \ldots z_{b}}{z_{1} \ldots z_{b+k}} y_{0}
$$

As a consequence, we see that $\mathcal{N}_{\vec{a}} \subset \mathbb{C}^{3 n}$ contains a copy of $\left(\mathbb{C}^{*}\right)^{n+2}$ as an open subset, with an action of $\left(\mathbb{C}^{*}\right)^{n+2}$ on $\mathcal{N}_{\vec{a}}$ which restricts to the usual action by multiplication on that subset. This is one of the standard definitions of a toric variety (see [7, 31, 32] for an introduction). In fact, since $\mathcal{N}_{\vec{a}}$ is a subvariety of $\mathbb{C}^{3 n}$ defined by monomial relations, it is an "affine toric variety," which is somewhat simpler than the general case.

Toric geometry describes a space by the well-defined algebraic functions it supports. Any monomial in the coordinates $x_{i}, y_{i}$ and $z_{i}$ defines such a function on $\mathcal{N}_{\vec{a}}$, but they are not all independent - as we have seen, we can use (4.2) and (4.3) to express $2 n-2$ of the basic coordinate functions in terms of $x_{0}, y_{0}$ and $z_{i}$. Changing notation temporarily, we let $\left(v_{-2}, v_{-1}, v_{0}, \ldots, v_{n-1}\right)=\left(x_{0}, y_{0}, z_{0}, \ldots, z_{n-1}\right)$ and $\left(w_{0}, \ldots, w_{3 n-1}\right)=$ $\left(x_{0}, \ldots, x_{n-1}, y_{0}, \ldots, y_{n-1}, z_{0}, \ldots, z_{n-1}\right)$. Then we can write $w_{i}=\prod v_{j}^{m_{i j}}$ and regard each 
$\vec{m}_{i} \in M=\mathbb{Z}^{n+2}$ as a vector of exponents. A general monomial will now be associated with a point in $M$ given by a sum of these basis vectors with non-negative integer coefficients. The set of all such points will form a cone $M_{+} \subset M$.

Conversely, a basis for the algebra of functions on $\mathcal{N}_{\vec{a}}$ determines the space $\mathcal{N}_{\vec{a}}$. The cone $M_{+}$determines such an algebra and basis - each point in the cone gives us a basis vector, and the multiplication law is induced from addition in $\mathbb{Z}^{n+2}$.

Since we can reconstruct $\mathcal{N}_{\vec{a}}$ from the cone $M_{+}$, we can use any description of the cone to define the space. Another description is in terms of a set of hyperplanes which bound the cone, i.e., vectors $\vec{n}$ for which

$$
\vec{n} \cdot \vec{m} \geq 0
$$

These vectors are points in the dual lattice $N \cong \operatorname{Hom}(M, \mathbb{Z})$. Clearly the sum of two solutions $\vec{n}$ of (4.4) will also satisfy (4.4) for all $M_{+}$, so this condition defines a dual cone $N_{+} \subset N$. Given $N_{+}$, the original cone $M_{+}$can be reconstructed, so the space $\mathcal{N}_{\vec{a}}$ can also be specified by a choice of cone $N_{+}$.

The advantage of the cone $N_{+}$is that it allows us to give an alternate description of the toric variety $\mathcal{N}_{\vec{a}}$ as a quotient. There are two approaches to presenting a toric variety as a quotient [33,34 - it can be thought of as a holomorphic quotient, or (if Kähler) as a symplectic quotient. (The relationship between the two is discussed in some detail in [15,16 to which the reader can refer for more details.) In a nutshell, a toric variety of dimension $k$ can be expressed in the form $\left(\mathbb{C}^{q}-F_{\Delta}\right) /\left(\mathbb{C}^{*}\right)^{q-k}$ for some set $F_{\Delta}$ and some $\left(\mathbb{C}^{*}\right)^{q-k}$ action in $\mathbb{C}^{q}$. The latter action can be carried out as written (the holomorphic quotient) or (in the Kähler case) it can be carried out in two steps, an $\left(\mathbb{R}_{+}\right)^{q-k}$ action and a $U(1)^{q-k}$ action (the symplectic quotient). The resulting quotient space depends on the precise form of the gauge fixing determined by the $\left(\mathbb{R}_{+}\right)^{q-k}$ action, the so-called moment map. The distinct possibilities, in fact, are the phases of [7, 8]. In the holomorphic approach, these phases are distinguished by being associated to different triangulations of certain point sets which in turn determine different point sets $F_{\Delta}$ to be removed from the initial parent space. If all possibilities are considered - that is, all triangulations and all gauge fixings - then all phases are accessed. This is the case for perturbative closed string theory. If, however, only a subset of gauge fixings are relevant for the physical model, then only some of the phases are physically realized. This turns out to be the case for D-branes. 
To implement this quotient description, we introduce homogeneous coordinates $p_{i}, i=$ $0, \ldots, q-1$ in the sense of [35], associated to a set $\mathcal{A}$ consisting of $q$ points in the lattice $N$. Taking integer linear combinations of these points defines a natural map

$$
T: \mathbb{Z}^{\mathcal{A}} \rightarrow N
$$

which we assume to be surjective. The transpose of the kernel of $T$ is then a $(q-k) \times k$ "charge matrix" $Q$, which specifies an action of $U(1)^{q-k}\left(\right.$ or $\left.\left(\mathbb{C}^{*}\right)^{q-k}\right)$ on $\mathbb{C}^{q}$. A triangulation $\Delta$ of the convex hull of $\mathcal{A}$ then determines - through a specific combinatorial procedure - a subset $F_{\Delta} \subset \mathbb{C}^{q}$ such that the toric variety takes the form $\left(\mathbb{C}^{q}-F_{\Delta}\right) /\left(\mathbb{C}^{*}\right)^{q-k}$. (See [7] for the combinatorial details of this step.) In the case of the toric variety $\mathcal{N}_{\vec{a}}$, the point set $\mathcal{A}$ consists of the generators of the cone $N_{+}$.

For our present purposes, we also need to see how the action of $\left(\mathbb{C}^{*}\right)^{k}$ on the toric variety (which was part of its definition) is represented in these terms. Since $\mathbb{C}^{q}-F_{\Delta}$ always contains $\left(\mathbb{C}^{*}\right)^{q}$, our toric variety $\left(\mathbb{C}^{q}-F_{\Delta}\right) /\left(\mathbb{C}^{*}\right)^{q-k}$ contains $\left(\mathbb{C}^{*}\right)^{q} /\left(\mathbb{C}^{*}\right)^{q-k} \cong\left(\mathbb{C}^{*}\right)^{k}$ as an open subset. That group will act on the toric variety through an action of $\left(\mathbb{C}^{*}\right)^{k}$ on $\mathbb{C}^{q}$, provided that the projection of that action to $\left(\mathbb{C}^{*}\right)^{q} /\left(\mathbb{C}^{*}\right)^{q-k} \cong\left(\mathbb{C}^{*}\right)^{k}$ gives the identity map. Such an action is specified by a $k \times q$ charge matrix $U$, and the condition on the projection is that

$$
T \cdot{ }^{t} U=\operatorname{Id}_{k},
$$

where $T$ is the $k \times q$ matrix (4.5) specifying the toric variety. We choose such a matrix $U$.

From the point of view of Witten's gauged linear sigma model, this mathematical construction can be physically realized by interpreting the $p_{i}$ as chiral superfields interacting in a $U(1)^{q-k}$ two-dimensional gauged linear sigma model. Inclusion of the Fayet-Iliopoulos D-terms with coefficients $\xi_{j}, j=1, \ldots, q-k$ provides the degrees of freedom which are the counterparts to the possible triangulations of the $p_{i}$. It is by now well known that the formulation of a toric variety using the holomorphic quotient of the last paragraph is equivalent to the Witten's physical incarnation since the latter realizes the same toric variety as a symplectic quotient $\mathbb{C}^{q} / / U(1)^{q-k}$. So, given a D-brane configuration, we can follow the procedure of this section to generate mathematical toric data which can then be re-translated into the physics of a gauged linear sigma model.

From a topological point of view, if the $\xi_{j}$ are completely generic, then all possible phases of the linear sigma model are physically realized, corresponding to all $c_{1}=0$ birational transformations on the toric variety $V$. That is, backgrounds corresponding to all 
possible triangulations of the point set $\mathcal{A}$ are realized. If, on the contrary, only a restricted set of values for the $\xi_{j}$ are physical, then only a subset of the possible phases will be probed. This is precisely what happens in constructing the D-brane moduli space. Namely, as we shall see below in the context of two examples, the D-brane vacuum moduli space on $\mathbb{C}^{3} / \mathbb{Z}_{n}$ gives precisely the standard toric point set $\mathcal{A}$ for the blow-up of $\mathbb{C}^{3} / \mathbb{Z}_{n}$. However, the $\xi_{i}$ parameters, determined by the $\zeta_{j}$ from the last section, are not generic and therefore only part of the phase space of the blown-up orbifold is probed by D-branes. Although we do not have a general proof, in the examples we have studied (and in examples studied by Sardo Infirri [6]) the phases which are eliminated are non-geometric phases reviewed earlier. This dovetails nicely with the result of Witten in [14] in which he found that the non-geometric phases are the ones which get squeezed out when passing to strongly coupled type IIA string theory - that is, M-theory. It is in this limit that D0-branes dominate low energy physics, and hence the fact that their vacuum moduli space - which we interpret as an effective spacetime background - does not see the non-geometric phases is in agreement with Witten's result.

To see this explicitly, we follow the procedure outlined above. Namely, we express the solution to the superpotential constraint in terms of a cone $M_{+}$. We take its dual to get a cone $N_{+}$. The $q$ generators of $N_{+}$determine a map $T: \mathbb{Z}^{\mathcal{A}} \rightarrow N$, and the transpose of the kernel of $T$ determines a $(q-n-2) \times q$ charge matrix $Q$. In order to obtain $\mathcal{N}_{\vec{a}}$ as the symplectic quotient $\mathbb{C}^{q} / / U(1)^{q-n-2}$, we must set the associated D-terms $\xi_{1}, \ldots$, $\xi_{q-n-2}$ to zero. To describe the action of the $U(1)^{n-1}$ charges in the twisted sectors on $\mathcal{N}_{\vec{a}}$, we represent the action of $U(1)^{n-1}$ on $\mathbb{C}^{n+2}$ by an $(n-1) \times(n+2)$ charge matrix $V$; the product $V U$ will then give an $(n-1) \times q$ charge matrix for the action of $U(1)^{n-1}$ on $\mathbb{C}^{q}$. (This charge matrix depends on the choice of a matrix $U$ satisfying (4.6), but different choices will only differ by charges from the matrix $Q$.) Non-zero values of the corresponding D-terms $\xi_{i}=\zeta_{i-(q-n-2)}$ are allowed.

The full set of charges is now given by a $(q-3) \times q$ charge matrix $\widetilde{Q}$, the concatenation of $Q$ and $V U$. The cokernel of its transpose gives toric data for the D-brane vacuum moduli space, in the form of a map $\widetilde{T}: \mathbb{Z}^{\mathcal{A}} \rightarrow \mathbb{Z}^{3}$. We claim that this data is nothing but the usual toric data for resolving the quotient space $\mathbb{C}^{3} / \mathbb{Z}_{n}$ (supplemented by some extra auxiliary fields as well as some extra $U(1)$ 's which can be used to eliminate them) showing an explicit realization of the D-brane moduli space aligning with the underlying spacetime structure. Second, we also claim that the non-genericity of the Fayet-Iliopoulos parameters is such that the non-geometric phases of this desingularization are not physically realized. 
We start with the case $n=3$. Following the procedure outlined, the cone $M_{+}$is generated by the rows in the following:

$\begin{array}{cccccc} & x_{0} & y_{0} & z_{0} & z_{1} & z_{2} \\ x_{0} & 1 & 0 & 0 & 0 & 0 \\ x_{1} & 1 & 0 & -1 & 1 & 0 \\ x_{2} & 1 & 0 & -1 & 0 & 1 \\ y_{0} & 0 & 1 & 0 & 0 & 0 \\ y_{1} & 0 & 1 & -1 & 1 & 0 \\ y_{2} & 0 & 1 & -1 & 0 & 1 \\ z_{0} & 0 & 0 & 1 & 0 & 0 \\ z_{1} & 0 & 0 & 0 & 1 & 0 \\ z_{2} & 0 & 0 & 0 & 0 & 1\end{array}$

and the charges of the fields $\left(x_{0}, y_{0}, z_{0}, \ldots, z_{2}\right)$ under the twisted sector gauge group $U(1)^{2}$ (with D-terms $\zeta_{1}, \zeta_{2}$, as in (3.4)) are given by

$$
V=\left(\begin{array}{ccccc}
0 & 0 & 0 & -1 & 1 \\
1 & 1 & 1 & 0 & -1
\end{array}\right)
$$

The dual cone $N_{+}$is generated by the columns of

$$
T=\left(\begin{array}{llllll}
1 & 0 & 0 & 1 & 0 & 0 \\
0 & 1 & 0 & 1 & 0 & 0 \\
0 & 0 & 1 & 1 & 0 & 0 \\
0 & 0 & 1 & 0 & 1 & 0 \\
0 & 0 & 1 & 0 & 0 & 1
\end{array}\right)
$$

(which correspond to homogeneous coordinates $p_{0}, \ldots, p_{5}$ ). The columns of $T$ were found as solutions of the equations (4.4), with the monomial corresponding to the hyperplane $\vec{n}$ being $\prod X_{i}^{\vec{n} \cdot \vec{m}_{i}}$.

The transpose of the kernel of this matrix is

$$
Q=\left(\begin{array}{llllll}
1 & 1 & 1 & -1 & -1 & -1
\end{array}\right)
$$

We choose $U$ satisfying (4.6) to be

$$
U=\left(\begin{array}{cccccc}
0 & -1 & -1 & 1 & 1 & 1 \\
0 & 1 & 0 & 0 & 0 & 0 \\
0 & 0 & 1 & 0 & -1 & -1 \\
0 & 0 & 0 & 0 & 1 & 0 \\
0 & 0 & 0 & 0 & 0 & 1
\end{array}\right)
$$


so that the full charge matrix $\widetilde{Q}$ obtained by concatenating $Q$ and $V U$ is

$$
\widetilde{Q}=\left(\begin{array}{cccccc}
1 & 1 & 1 & -1 & -1 & -1 \\
0 & 0 & 0 & 0 & -1 & 1 \\
0 & 0 & 0 & 1 & 0 & -1
\end{array}\right)
$$

The cokernel of the transpose of $\widetilde{Q}$ (which can be calculated as the transpose of the kernel) is the matrix

$$
\left(\begin{array}{cccccc}
-1 & 1 & 0 & 0 & 0 & 0 \\
-1 & 0 & 1 & 0 & 0 & 0 \\
3 & 0 & 0 & 1 & 1 & 1
\end{array}\right)
$$

This matrix has three identical columns, which means that the point set $\widetilde{\mathcal{A}}$ determined by those columns actually only contains four distinct elements. If we eliminate the redundant variables and $U(1)$ 's, we obtain a smaller matrix

$$
\widetilde{T}=\left(\begin{array}{cccc}
-1 & 1 & 0 & 0 \\
-1 & 0 & 1 & 0 \\
3 & 0 & 0 & 1
\end{array}\right)
$$

specifying the toric data for the D-brane moduli space $\mathcal{N}_{\vec{a}} / / U(1)^{n-1}$.

We recognize this as the toric data for resolving $\mathbb{C}^{3} / \mathbb{Z}_{3}$. (The charge matrix determined by $\widetilde{T}$ is $\left(\begin{array}{llll}1 & 1 & 1 & -3\end{array}\right)$.) Triangulating on all points yields the blow-up $\mathcal{O}(-3) \rightarrow \mathbb{P}^{2}$. Leaving out the central point, in the context of type II string theory, would take us to the Landau-Ginsburg phase, which is geometrically associated with the blown-down singular variety. In Witten's gauged linear sigma model, these phases arise from studying the bosonic potential

$$
U=\left(\left|u_{1}\right|^{2}+\left|u_{2}\right|^{2}+\left|u_{3}\right|^{2}-3|p|^{2}-\xi\right)^{2}
$$

for $\xi$ positive and for $\xi$ negative. In the former case, vanishing of the potential is consistent with $p=0$, which directly gives the exceptional divisor, $\mathbb{P}^{2}$ with size controlled by $\xi$. When $\xi$ is negative, though, $p$ cannot vanish, corresponding to the exceptional divisor being blown down.

In our case, we have precisely the same toric point set (the columns of (4.14)) but the Fayet-Iliopoulos parameters $\zeta_{i}$ are only associated with the last two $U(1)$ quotients in (4.12) since the first actually originates from a superpotential constraint in the original D-brane action. As $\zeta_{1}$ and $\zeta_{2}$ take on generic values, it is not hard to see that the corresponding value of $\xi$ is non-negative, thereby only accessing the blown-up phase. To see this note that for any non-zero values of the $\zeta_{i}$, the second two D-terms imply that two out of the last 
three homogeneous variables $p_{3}, p_{4}, p_{5}$ are nonzero. Calling the one that can potentially vanish $\tilde{p}$, we can solve the latter two D-term constraints in terms of $\tilde{p}$ and therefore rewrite the first D-term as

$$
\left(\left|p_{0}\right|^{2}+\left|p_{1}\right|^{2}+\left|p_{2}\right|^{2}-3|\tilde{p}|^{2}-f\left(\zeta_{1}, \zeta_{2}\right)\right)^{2} .
$$

We claim that $f\left(\zeta_{1}, \zeta_{2}\right)>0$. In fact, if we write the charge matrix again with an extra column including the D-terms

$$
\left(\begin{array}{ccccccc}
1 & 1 & 1 & -1 & -1 & -1 & 0 \\
0 & 0 & 0 & 0 & -1 & 1 & \zeta_{1} \\
0 & 0 & 0 & 1 & 0 & -1 & \zeta_{2}
\end{array}\right)
$$

and then modify that augmented matrix by invertible row operations to

$$
\left(\begin{array}{ccccccc}
1 & 1 & 1 & 0 & 0 & -3 & -\zeta_{1}+\zeta_{2} \\
0 & 0 & 0 & 0 & 1 & -1 & -\zeta_{1} \\
0 & 0 & 0 & 1 & 0 & -1 & \zeta_{2}
\end{array}\right)
$$

we see that when $\zeta_{1}<0$ and $\zeta_{2}>0$, we have $p_{4} \neq 0$ and $p_{3} \neq 0$, and also $f\left(\zeta_{1}, \zeta_{2}\right)=$ $-\zeta_{1}+\zeta_{2}>0$. This verifies our claim in one of three phase regions, illustrated in figure 2 . Similar verifications can be made in the other two regions, using the alternate forms

$$
\left(\begin{array}{ccccccc}
1 & 1 & 1 & -3 & 0 & 0 & -\zeta_{1}-2 \zeta_{2} \\
0 & 0 & 0 & -1 & 1 & 0 & -\zeta_{1}-\zeta_{2} \\
0 & 0 & 0 & -1 & 0 & 1 & -\zeta_{2}
\end{array}\right) \text { and }\left(\begin{array}{ccccccc}
1 & 1 & 1 & 0 & -3 & 0 & 2 \zeta_{1}+\zeta_{2} \\
0 & 0 & 0 & 0 & -1 & 1 & \zeta_{1} \\
0 & 0 & 0 & 1 & -1 & 0 & \zeta_{1}+\zeta_{2}
\end{array}\right)
$$

of (4.17).

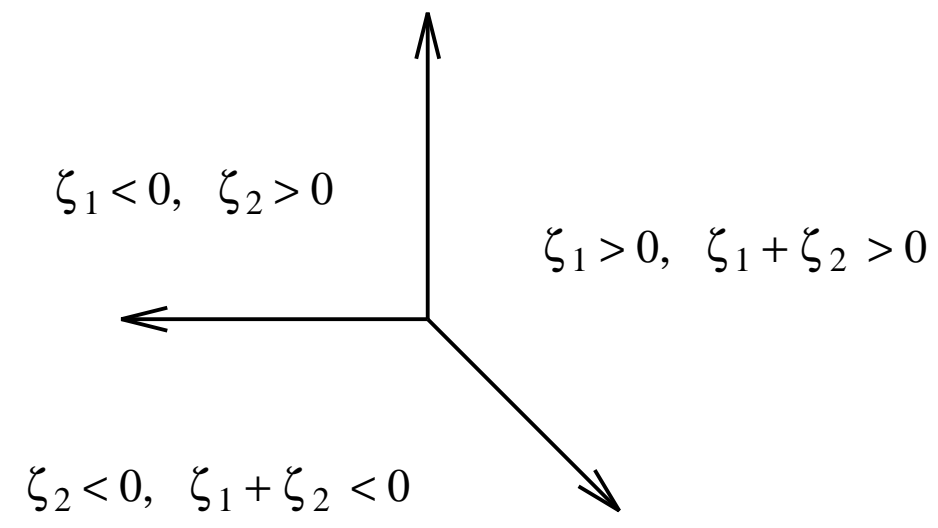

Fig. 2: Phases in the $\left(\zeta_{1}, \zeta_{2}\right)$ plane. 
The case of $n=5$ is similar, but technically more involved. Taking the action to be $\left(Z^{1}, Z^{2}, Z^{3}\right) \rightarrow\left(\omega^{3} Z^{1}, \omega^{3} Z^{2}, \omega^{-1} Z^{3}\right)$, we find that the cone $M_{+}$is generated by the rows in the following:

$\begin{array}{cccccccc} & x_{0} & y_{0} & z_{0} & z_{1} & z_{2} & z_{3} & z_{4} \\ x_{0} & 1 & 0 & 0 & 0 & 0 & 0 & 0 \\ x_{1} & 1 & 0 & 0 & 1 & 0 & 0 & -1 \\ x_{2} & 1 & 0 & -1 & 1 & 1 & 0 & -1 \\ x_{3} & 1 & 0 & -1 & 0 & 1 & 1 & -1 \\ x_{4} & 1 & 0 & -1 & 0 & 0 & 1 & 0 \\ y_{0} & 0 & 1 & 0 & 0 & 0 & 0 & 0 \\ y_{1} & 0 & 1 & 0 & 1 & 0 & 0 & -1 \\ y_{2} & 0 & 1 & -1 & 1 & 1 & 0 & -1 \\ y_{3} & 0 & 1 & -1 & 0 & 1 & 1 & -1 \\ y_{4} & 0 & 1 & -1 & 0 & 0 & 1 & 0 \\ z_{0} & 0 & 0 & 1 & 0 & 0 & 0 & 0 \\ z_{1} & 0 & 0 & 0 & 1 & 0 & 0 & 0 \\ z_{2} & 0 & 0 & 0 & 0 & 1 & 0 & 0 \\ z_{3} & 0 & 0 & 0 & 0 & 0 & 1 & 0 \\ z_{4} & 0 & 0 & 0 & 0 & 0 & 0 & 1,\end{array}$

and the charges of the fields $\left(x_{0}, y_{0}, z_{0}, \ldots, z_{4}\right)$ under the twisted sector gauge group $U(1)^{4}$ (with D-terms $\zeta_{1}, \ldots, \zeta_{4}$, as in (3.4)) are given by

$$
V=\left(\begin{array}{ccccccc}
0 & 0 & 0 & -1 & 1 & 0 & 0 \\
0 & 0 & 0 & 0 & -1 & 1 & 0 \\
1 & 1 & 0 & 0 & 0 & -1 & 1 \\
0 & 0 & 1 & 0 & 0 & 0 & -1
\end{array}\right) .
$$

The dual cone $N_{+}$is generated by the columns of

$$
T=\left(\begin{array}{lllllllllllll}
1 & 0 & 0 & 1 & 0 & 0 & 0 & 0 & 1 & 0 & 0 & 0 & 1 \\
0 & 1 & 0 & 1 & 0 & 0 & 0 & 0 & 1 & 0 & 0 & 0 & 1 \\
0 & 0 & 1 & 1 & 0 & 0 & 1 & 1 & 1 & 0 & 0 & 0 & 0 \\
0 & 0 & 1 & 0 & 1 & 1 & 1 & 0 & 0 & 1 & 0 & 0 & 0 \\
0 & 0 & 1 & 1 & 1 & 0 & 0 & 1 & 0 & 0 & 1 & 0 & 0 \\
0 & 0 & 1 & 0 & 0 & 1 & 1 & 1 & 0 & 0 & 0 & 1 & 0 \\
0 & 0 & 1 & 1 & 1 & 1 & 0 & 0 & 0 & 0 & 0 & 0 & 1
\end{array}\right)
$$

(which correspond to homogeneous coordinates $p_{0}, \ldots, p_{12}$ ). We choose $U$ satisfying (4.6) to be

$$
U=\left(\begin{array}{ccccccccccccc}
1 & 0 & 0 & 0 & 0 & 0 & 0 & 0 & 0 & 0 & 0 & 0 & 0 \\
0 & 1 & 0 & 0 & 0 & 0 & 0 & 0 & 0 & 0 & 0 & 0 & 0 \\
-1 & -1 & 0 & 0 & 0 & 0 & 0 & 0 & 1 & 0 & 0 & 0 & 0 \\
0 & 0 & 0 & 0 & 0 & 0 & 0 & 0 & 0 & 1 & 0 & 0 & 0 \\
0 & 0 & 0 & 0 & 0 & 0 & 0 & 0 & 0 & 0 & 1 & 0 & 0 \\
0 & 0 & 0 & 0 & 0 & 0 & 0 & 0 & 0 & 0 & 0 & 1 & 0 \\
-1 & -1 & 0 & 0 & 0 & 0 & 0 & 0 & 0 & 0 & 0 & 0 & 1
\end{array}\right)
$$


so that the full charge matrix $\widetilde{Q}$ obtained by concatenating $Q={ }^{t}(\operatorname{ker} T)$ and $V U$ (and including an extra column for the D-terms) is

$$
\widetilde{Q}=\left(\begin{array}{cccccccccccccc}
1 & 1 & 0 & 2 & -1 & -1 & 2 & -1 & -3 & 0 & 0 & 0 & 0 & 0 \\
0 & 0 & 1 & -1 & 0 & 0 & -1 & 0 & 1 & 0 & 0 & 0 & 0 & 0 \\
0 & 0 & 0 & 0 & 0 & 0 & -1 & 1 & 0 & 1 & -1 & 0 & 0 & 0 \\
0 & 0 & 0 & 0 & -1 & 1 & 0 & 0 & 0 & 0 & 1 & -1 & 0 & 0 \\
0 & 0 & 0 & 1 & 0 & 0 & 0 & -1 & 0 & 0 & 0 & 1 & -1 & 0 \\
0 & 0 & 0 & 0 & 0 & -1 & 1 & 0 & -1 & 0 & 0 & 0 & 1 & 0 \\
0 & 0 & 0 & 0 & 0 & 0 & 0 & 0 & 0 & -1 & 1 & 0 & 0 & \zeta_{1} \\
0 & 0 & 0 & 0 & 0 & 0 & 0 & 0 & 0 & 0 & -1 & 1 & 0 & \zeta_{2} \\
0 & 0 & 0 & 0 & 0 & 0 & 0 & 0 & 0 & 0 & 0 & -1 & 1 & \zeta_{3} \\
0 & 0 & 0 & 0 & 0 & 0 & 0 & 0 & 1 & 0 & 0 & 0 & -1 & \zeta_{4}
\end{array}\right) .
$$

The cokernel of the transpose of $\widetilde{Q}$ (which can be calculated as the transpose of the kernel) is the matrix

$$
\left(\begin{array}{ccccccccccccc}
-1 & 1 & 0 & 0 & 0 & 0 & 0 & 0 & 0 & 0 & 0 & 0 & 0 \\
-3 & 0 & 1 & 0 & 0 & 0 & 0 & 0 & -1 & -1 & -1 & -1 & -1 \\
5 & 0 & 0 & 1 & 1 & 1 & 1 & 1 & 2 & 2 & 2 & 2 & 2
\end{array}\right)
$$

This matrix has two sets of identical columns (with five in each set), which means that the point set $\widetilde{\mathcal{A}}$ determined by the columns actually only contains five distinct elements. If we eliminate the redundant variables and $U(1)$ 's, we obtain a smaller matrix

$$
\widetilde{T}=\left(\begin{array}{ccccc}
-1 & 1 & 0 & 0 & 0 \\
-3 & 0 & 1 & 0 & -1 \\
5 & 0 & 0 & 1 & 2
\end{array}\right)
$$

specifying the toric data for the D-brane moduli space $\mathcal{N}_{\vec{a}} / / U(1)^{n-1}$. The corresponding charge matrix ${ }^{t}(\operatorname{ker} \widetilde{T})$ is

$$
\left(\begin{array}{ccccc}
1 & 1 & 0 & 1 & -3 \\
0 & 0 & 1 & -2 & 1
\end{array}\right)
$$

We recognize this toric data in $\mathbb{R}^{3}$ as that for $\mathbb{C}^{3} / \mathbb{Z}_{5}$. From the point of view of a symplectic quotient or the gauged linear sigma model, this corresponds to five chiral fields with bosonic potential

$$
U=\left(\left|u_{1}\right|^{2}+\left|u_{2}\right|^{2}+\left|u_{4}\right|^{2}-3|p|^{2}-\xi_{1}\right)^{2}+\left(\left|u_{3}\right|^{2}-2\left|u_{4}\right|^{2}+|p|^{2}-\xi_{2}\right)^{2}
$$

This linear sigma model has four phases, one of which is the (single) smooth geometrical resolution (represented by $\xi_{1}>0, \xi_{2}>0$ ), two of which are orbifold phases (in which 
one or other of the exceptional divisors has been blown-down) and a Landau-Ginsburg phase (where this terminology is more apt for models with a superpotential, but we retain the common labeling scheme). As before, if we had completely generic Fayet-Iliopoulos terms associated with each row of the full $U(1)^{10}$ charge matrix (4.24), then the D-brane moduli space would probe all four of these phases. But, since we only have non-zero $\zeta_{j}$ associated with the $U(1)^{4}$ twisted sector gauge group, this is not the case. Rather, the three "non-geometrical" phases which disappear in the strongly coupled type IIA string are not realized as the $\zeta$ 's take on all possible values: only the geometric phase is probed by the D-branes.

We will check this statement explicitly only in one phase of the model. By invertible row operations, we can modify (4.24) to

$$
\left(\begin{array}{cccccccccccccc}
1 & 1 & 0 & 0 & 0 & 0 & 1 & 0 & 0 & -3 & 0 & 0 & 0 & 2 \zeta_{1}+2 \zeta_{2}+\zeta_{3}+\zeta_{4} \\
0 & 0 & 1 & 0 & 0 & 0 & -2 & 0 & 0 & 1 & 0 & 0 & 0 & -\zeta_{2}-\zeta_{4} \\
0 & 0 & 0 & 0 & 0 & 0 & -1 & 1 & 0 & 0 & 0 & 0 & 0 & \zeta_{1} \\
0 & 0 & 0 & 0 & 1 & 0 & -1 & 0 & 0 & 0 & 0 & 0 & 0 & -\zeta_{2}-\zeta_{4} \\
0 & 0 & 0 & 1 & 0 & 0 & -1 & 0 & 0 & 0 & 0 & 0 & 0 & \zeta_{1}+\zeta_{3} \\
0 & 0 & 0 & 0 & 0 & 1 & -1 & 0 & 0 & 0 & 0 & 0 & 0 & -\zeta_{4} \\
0 & 0 & 0 & 0 & 0 & 0 & 0 & 0 & 0 & -1 & 1 & 0 & 0 & \zeta_{1} \\
0 & 0 & 0 & 0 & 0 & 0 & 0 & 0 & 0 & -1 & 0 & 1 & 0 & \zeta_{1}+\zeta_{2} \\
0 & 0 & 0 & 0 & 0 & 0 & 0 & 0 & 0 & -1 & 0 & 0 & 1 & \zeta_{1}+\zeta_{2}+\zeta_{3} \\
0 & 0 & 0 & 0 & 0 & 0 & 0 & 0 & 1 & -1 & 0 & 0 & 0 & \zeta_{1}+\zeta_{2}+\zeta_{3}+\zeta_{4}
\end{array}\right)
$$

From this form it becomes clear that if the $\zeta_{i}$ 's satisfy

$$
\begin{array}{r}
\zeta_{1}>0, \quad-\zeta_{2}-\zeta_{4}>0, \quad \zeta_{1}+\zeta_{3}>0, \quad-\zeta_{4}>0 \\
\zeta_{1}+\zeta_{2}>0, \quad \zeta_{1}+\zeta_{2}+\zeta_{3}>0, \quad \zeta_{1}+\zeta_{2}+\zeta_{3}+\zeta_{4}>0
\end{array}
$$

then none of $p_{3}, p_{4}, p_{5}, p_{7}, p_{8}, p_{10}, p_{11}$, or $p_{12}$ can vanish; moreover, $\xi_{1}=2 \zeta_{1}+2 \zeta_{2}+\zeta_{3}+\zeta_{4}$ and $\xi_{2}=-\zeta_{2}-\zeta_{4}$ are both positive under these conditions, so we end up in the geometric phase.

\section{Conclusions}

In this paper we have discussed the topological properties of D-brane vacuum moduli space in the context of abelian orbifolds $\mathbb{C}^{3} / \Gamma$ for $\Gamma \subset S U(3)$ and their smooth resolutions. We have found that these moduli spaces emerge as Kähler quotients with moment maps determined by Fayet-Iliopoulos parameters. Examination of these quotients shows that they 
are birational to the singular space $\mathbb{C}^{3} / \Gamma$ with the Fayet-Iliopoulos parameters controlling the size of the blow-up to a smooth space. This alignment of the vacuum moduli space and the ambient background space provides another strong piece of evidence that spatial backgrounds at ultrashort distances can be thought of as a derivative concept emerging from the fundamental notion of a vacuum moduli space.

The classical vacuum moduli space coincides with the moduli space of another theory, a gauged linear sigma model with no superpotential, motivated by considerations of toric geometry. 1 Hence, the moduli space can a priori have a rich phase structure. However, non-genericity of the Fayet-Iliopoulos parameters appears to ensure that the non-geometric phases are not part of the physical vacuum D-brane moduli space.

The neighborhood of an orbifold point can contain other transitions. For example, the $\mathbb{C}^{3} / \mathbb{Z}_{11}$ orbifold has multiple resolutions, connected by flops. This D-brane linear sigma model will contain an explicit geometric realization of the flop, and it will be interesting to work this out.

It is worth noting that even for $\Gamma \not \subset S U(3)$, the D-brane theory defined as a quotient of $\mathcal{N}=4$ super-Yang-Mills theory is a sensible non-supersymmetric gauge theory, whose classical moduli space (on the Higgs branch) is an ALE space asymptotic to $\mathbb{C}^{3} / \Gamma$, also studied in [5,6]. These are non-supersymmetric theories with special matter content, and it would be interesting to see if this moduli space survives in the quantum theory.

\section{Acknowledgments}

We thank the members of the Rutgers high energy theory group for discussions; B.R.G. and D.R.M. also thank the Rutgers group for hospitality during various stages of this project. The work of M.R.D. is supported by DOE grant DE-FG02-96ER40959. The work of B.R.G. is supported by a National Young Investigator Award and the Alfred P. Sloan Foundation. The work of D.R.M. is supported in part by the Harmon Duncombe Foundation and by NSF grants DMS-9401447 and DMS-9627351.

4 It would be interesting to relate the D-brane worldvolume theory to this other theory more directly, perhaps by means of a duality transformation in which the massive states associated to "fractional branes" play a role. 


\section{References}

[1] M. R. Douglas and G. Moore, "D-Branes, Quivers, and ALE Instantons," hepth/9603167.

[2] J. Polchinski, "Tensors from K3 Orientifolds," hep-th/9606165.

[3] C. Johnson and R. Myers, "Aspects of Type IIB Theory on ALE Spaces," hepth/9610140.

[4] P. B. Kronheimer, "The Construction of ALE Spaces as Hyper-Kähler Quotients," J. Diff. Geom. 29 (1989) 665.

[5] A. V. Sardo Infirri, "Partial Resolutions of Orbifold Singularities via Moduli Spaces of HYM-type Bundles," alg-geom/9610004.

[6] A. V. Sardo Infirri, "Resolutions of Orbifold Singularities and Flows on the McKay Quiver," alg-geom/9610005.

[7] P. S. Aspinwall, B. R. Greene and D. R. Morrison, "Calabi-Yau Moduli Space, Mirror Manifolds and Spacetime Topology Change in String Theory," Nucl. Phys. B416 (1994) 414; hep-th/9309097.

[8] E. Witten, "Phases of $N=2$ Theories In Two Dimensions," Nucl. Phys. B403 (1993) 159; hep-th/9301042.

[9] P. S. Aspinwall, B. R. Greene and D. R. Morrison, "Measuring Small Distances in $N=2$ Sigma Models," Nucl. Phys. B420 (1994) 184; hep-th/9311042.

[10] T. Banks, W. Fischler, S. H. Shenker and L. Susskind, "M Theory as a Matrix Model: A Conjecture," hep-th/9610043.

[11] M. R. Douglas, "Enhanced Gauge Symmetry in M(atrix) Theory," hep-th/9612126.

[12] M. R. Douglas, H. Ooguri and S. H. Shenker, "Issues in M(atrix) Theory Compactification," hep-th/9702203.

[13] M. R. Douglas and B. R. Greene, to appear.

[14] E. Witten, "Phase Transitions In M-Theory And F-Theory," Nucl. Phys. B471 (1996) 195; hep-th/9603150.

[15] P. S. Aspinwall and B. R. Greene, "On the Geometric Interpretation of $N=2$ Superconformal Theories," Nucl. Phys. B437 (1995) 205; hep-th/9409110.

[16] D. R. Morrison and M. R. Plesser, "Summing the Instantons: Quantum Cohomology and Mirror Symmetry in Toric Varieties," Nucl. Phys. B440 (1995) 279; hepth/9412236.

[17] P. S. Aspinwall, "Enhanced Gauge Symmetries and K3 Surfaces," Phys. Lett. B357 (1995) 329; hep-th/9507012.

[18] O. J. Ganor, D. R. Morrison, and N. Seiberg, "Branes, Calabi-Yau Spaces, and Toroidal Compactification of the $N=1$ Six-Dimensional $E_{8}$ Theory," Nucl. Phys. B487 (1997) 93 ; hep-th/9610251.

[19] D. R. Morrison and M. R. Plesser, to appear. 
[20] L. Dixon, J. A. Harvey, C. Vafa, and E. Witten, "Strings on Orbifolds, I, II" Nucl. Phys. B261 (1985) 678; Nucl. Phys. B274 (1986) 285.

[21] J. Polchinski and Y. Cai, "Consistency of Open Superstring Theories," Nucl. Phys. B296 (1988) 91.

[22] M. R. Douglas, "Branes within Branes," hep-th/9512077.

[23] H. Ooguri, Y. Oz and Z. Yin, "D-Branes on Calabi-Yau Spaces and Their Mirrors," Nucl.Phys. B477 (1996) 407; hep-th/9606112.

[24] E. G. Gimon and J. Polchinski, "Consistency Conditions for Orientifolds and DManifolds," Phys. Rev. D54 (1996) 1667; hep-th/9601038.

[25] A. Sagnotti, "Some Properties of Open-String Theories," hep-th/9509080.

[26] Y. Ito and M. Reid, "The McKay Correspondence for Finite Subgroups of SL(3,C)," in: Higher Dimensional Complex Varieties (M. Andreatta et al., eds.), de Gruyter, 1996, p. 221; alg-geom/9411010.

[27] M. Reid, "McKay Correspondence," alg-geom/9702016.

[28] B. R. Greene and Y. Kanter, "Small Volumes in Compactified String Theory," hepth/9612181.

[29] T. Banks, N. Seiberg, and E. Silverstein, "Zero and One-dimensional Probes with $N=8$ Supersymmetry," hep-th/9703052.

[30] M. R. Douglas, to appear.

[31] T. Oda, Convex Bodies and Algebraic Geometry, Springer-Verlag, 1988.

[32] W. Fulton, Introduction to Toric Varieties, Princeton University Press, 1993.

[33] T. Delzant, "Hamiltoniens périodiques et images convexe de l'application moment," Bull. Soc. Math. France 116 (1988) 315.

[34] M. Audin, The Topology of Torus Actions on Symplectic Manifolds, Birkhäuser, 1991.

[35] D. A. Cox, "The Homogeneous Coordinate Ring of a Toric Variety," J. Algebraic Geom. 4 (1995) 17; alg-geom/9210008. 